\title{
Rationale Use of Neutrophil-to-lymphocyte ratio for early diagnosis and stratification of COVID-19
}

\author{
Zahorec $\mathrm{R}^{1}$, Hulin $\mathrm{I}^{2}$, Zahorec $\mathrm{P}^{3}$ \\ Department of Anesthesiology and Intensive Medicine, St Elizabeths Cancer Institute and Medical School, \\ Comenius University, Bratislava, Slovakia. rzahorec@ousa.sk
}

\begin{abstract}
Coronavirus disease is caused by a virus that is the cause of a potentially fatal disease worldwide. Coronavirus is a pathogen that primarily affects the human respiratory system. Coronavirus 2019 (COVID-19) has been named WHO since February 11, 2020. The first cases of COVID-19 were reported in December 2019. In January 2020, COVID-19 infection was identified in hospitalized patients in Wuhan, China.

We analyze the role of neutrophil-lymphocyte ratio (NLR) in viral infection with special emphasize on novel corona virus disease-COVID-19. NLR may be used for early detection and may reflect progression to the more severe illness leading to SARS-CoV-2. In the mini review we investigate the use of NLR as a surrogate marker for diagnosis and stratification of COVID-19.

Clinical symptoms such as pneumonia, acute respiratory distress syndrome, acute heart damage have led to death. In some cases, multiple inflammations have been observed. Treatment with interferon inhalation showed no clinical effect and the condition worsened instead (Tab. 5, Fig. 1, Ref. 18). Text in PDF www.elis.sk KEY WORDS: neutrophil-to-lymphocyte ratio, corona virus SARS-CoV-2, COVID-19.
\end{abstract}

\section{Introduction}

Corona virus Disease 2019 (COVID-19) is spreading world wire. This pandemic infection of respiratory system is caused by novel coronavirus, severe acute respiratory syndrome coronavirus 2 (SARS-CoV-2). The clinical spectrum of SARS-CoV-2 infection appears wide, encompassing asympotmatic infection, mild upper respiratory tract illness, and severe viral pneumonia with respiratory failure, developing ARDS, multiorgan dysfunction, shock and even death (Zhou et al, 2020).

Recently retrospective studies were issued analyzing the clinical course and risk factors for mortality of adult in patients with COVID-19. Clinical, demographic, epidemiological and laboratory data may provide a valuable information set for early diagnosis, stratification and prognosis of COVID-19 (Zhou et al, 2020, Song, 2020, Liu et al, 2020).

Leading symptoms and signs of new coronavirus infection are: fatigue, myalgia, fever $37.5-38.3{ }^{\circ} \mathrm{C}$, cough, sometimes abdominal algia and diarrhoe. According clinical severity we recognize four stages:

${ }^{1}$ Department of Anesthesiology and Intensive Medicine. St Elizabeth s Cancer Institute and Medical school, Comenius University, Bratislava, Slovakia. ${ }^{2}$ Faculty of Medicine, Comenius University, Bratislava, Slovakia, and ${ }^{3}$ Faculty of Medicine, Comenius University, Bratislava, Slovakia

Address for correspondence: Roman Zahorec, MD, PhD, Department of Anesthesiology and Intensive Medicine. St Elizabeth s Cancer Institute and Medical school, Comenius University, Heydukova 10, SK-812 50 Bratislava, Slovakia.
1) mild - patients had no pneumonia on imaging,:

2) moderate: patients with symptoms and imaging examination show pneumonia,

3) severe COVID patients develope severe pneumonia with dyspnoe, and

a) respiratory rate more than $24-30$ breaths/minute,

b) resting pulse oxygen saturation less $\mathrm{SpO} 2<93 \%$,

c) oxygenation index $\mathrm{PaO}_{2} / \mathrm{FiO}_{2}<300 \mathrm{mmHg}$,

d) on chest X-ray progression more than $50 \%$ of multiple pulmonary lobes,

4) most severe cases:

a) critical patients with acute respiratory failure requiring mechanical ventilation,

b) shock,

c) other organ dysfunction developing multiorgan dysfunction syndrome (Tab.1).

Currently the diagnosis of COVID-19 relies mainly on SARS-CoV-2 nucleic acid detection. Time delay for taking specimen, relative shortage of detection kits and shortcomings of falsenegative results due to the low viral load in the samples may cause that many patients can not be detected in time (Song et al, 2020). Other problems are atypical early clinical symptoms, unclear epidemiological history. (Yang et al, 2020). Physicians are searching for easy available and reliable parameters for the screening and early diagnosis of COVID-19. Few articles on this topic were issued recently (Zhou et al, 2020, Song et al, 2020, Liu, 2020, Fu et al, 2020, Ma et al, 2020). 
Tab. 1. Clinical signs and symptoms of four stages of COVID-19 infection according clinical severity.

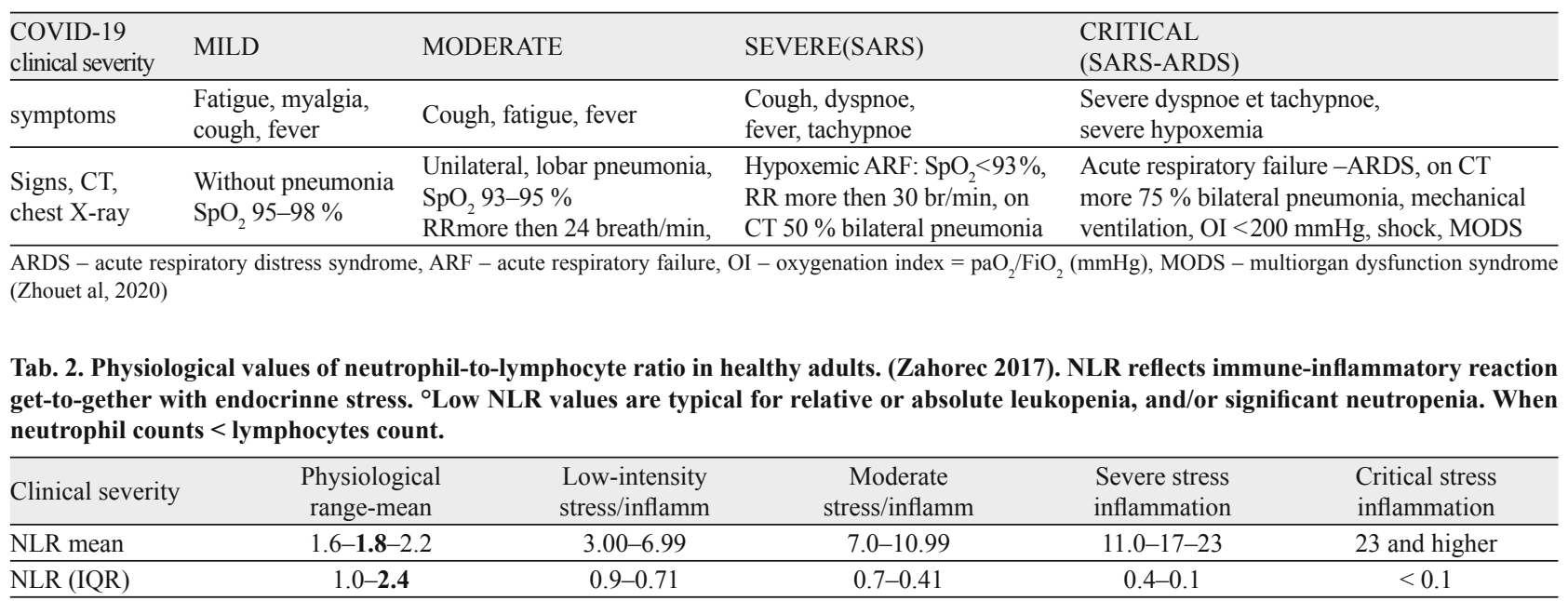

We focuse on blood investigation with special emphasize on differential white blood cell count (neutrophil-to-lymphocyte count, NLR) and complete blood count which may be helpful for screening and support the early diagnosis of COVID-19. Some valid laboratory parameters together with clinical symptoms, epidemiological hazard and imaging methods /(chest X-ray, CT) may behelpful to put right diagnosis or high-grade suspicion for COVID-19. Neutrophil-to-lymphocyte ratio (NLR) is simple, available and reliable parameter of immune-inflammatory response, neuroendocrinne stress and severity of illness (Fig.1). It is a very sensitive but less specific hematologic parameter to measure stress, intensity of infection/inflammation and severity of illness of various origin (Zahorec, 2001, 2017).

NLR reflects not only immune-inflammatory response, but even activity of vegetative nervous system (VNS). The sympathetic nervous system (SNS), innervates lymphoid organs and secreted hormones to provide the relationship between the brain

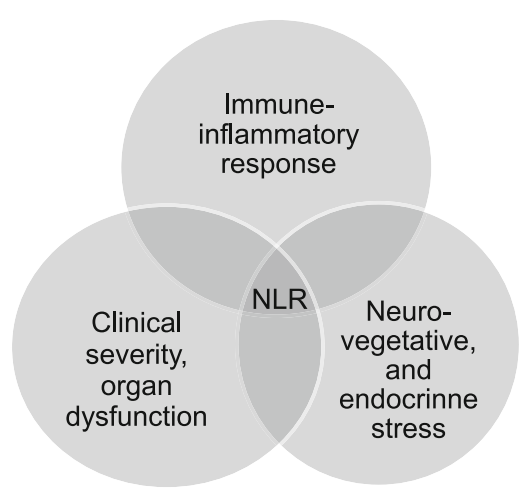

Fig. 1. The immunopathology and ethiology of dynamic changes of neutrophil-to-lymphocyte ratio regarding the impact of three suprasystems of human body: neurovegetative, neuroendocrinne, and immune system. and immune system. Two major populations of leukocytes (granulocytes and lymphocytes), are affected by activity of vegetative nervous system. Neutrophils are activated in number and function through sympathetic activationand stress hormones (catecholamines), whereas parasympathetic stimulation and hormones like cortizol modulate the function and redistribution of lymphocytes, which leads to the decrease of lymphocytes count in peripheral blood. The activation of the sympathetic nervous system triggers leukocytosis with neutrophilia, which also means an increment in neutrophil-lymphocyte ratio (Kalelioglu. 2019 Reiske et al, 2020).

Although high NLR rates in psychiatric disorders are considered to reflect an inflammatory status, it may refer an indirect reflection of the VNS's imbalance in favor of symphatetic activity. Therefore, NLR can be interpreted not only as a marker of inflammation, but also as a marker of stress (Fig. 1) and/or increased sympathetic activation Infusion of physiological doses of stress hormones (catecholamines and cortisol) to experimental animals (male pigs) induces changes in number and function of immune cells. All stress hormones promote innate over adaptive immune response. Catecholamines stimulates mainly increase number of circulating neutrophils ( neutrofília) and infusion of cortisol induces decrease number of cirkulatng lymphocytes (lymphopenia). Cortisol strongly decrease porcine adaptive immune cells and increase neutrophils (Reiske et al, 2020, Kalelioglu, 2019). This new interpretation of increase of NLR values may explain many phenomena in clinical medicine including critical illness, response to mutliple trauma, acute pancreatitis and even to SARS - severe acute respiratory syndrome in COVID-19.

The goal of our paper is to evaluate the role and clinical utility of NLR for early diagnosis and stratification of COVID-19 together with other perspective laboratory parameters (hs-troponin, D-dimer and/or serum ferritin).

Patients infected with COVID-19 exhibited higher leukocyte counts, abnormal respiratory findings, and only mild increased plasma levels of pro-inflammatory cytokines. Patient sputum sometimes showed positive polymerase chain reactions for novel coro- 
Tab. 3. Four biomarkers of inflammation for differential diagnosis /detection of viral and bacterial infections.

\begin{tabular}{lcc}
\hline Parameter/Infection & VIRAL & BACTERIAL/SEPSIS \\
\hline IL-6 pg/ml & $\mathbf{1 0}-\mathbf{2 0}-30-50 \mathrm{pg} / \mathrm{ml}$ & $100-\mathbf{2 0 0}-\mathbf{4 0 0}-800 \mathrm{pg} / \mathrm{ml}$ \\
\hline C-reactive protein mg/l & $\mathbf{5}-\mathbf{2 0}-30 \mathrm{mg} / 1$ & $50-\mathbf{1 0 0}-\mathbf{2 0 0} \mathrm{mg} / 1$ \\
\hline Procalcitonin $\mu \mathrm{g} / 1$ & $0,06-0.1 \mu \mathrm{g} / 1$ & $0.4-\mathbf{1 . 0}-\mathbf{5 . 0}-10 \mu \mathrm{g} / 1$ \\
\hline Neutrophil-lymphocyte & $1.1-5.9$ & $7.0-11.0$ moderate \\
Ratio - NLR values & $0.3-0.9$ relative neutropenia & $11.0-23$ severe bacteremia \\
\hline
\end{tabular}

Other study investigated the role of NLR in discriminating between different adult patients groups hospitalized for fever in Bergen University Hospital. The cohort consists of 299 patients: 150 patients with bacterial infection supported by microbiology and serology (69 had pneumonia, 30 urinary infection and 27 had septicemia), 14 patients had viral infection, nine suffered from infectiuous mononucleosis. 66

navirus SARS-CoV-2. Laboratory studies have shown leukopenia with a leukocyte count of $2.91 \times 10^{9}$ cells $/ 1$, of which $70.0 \%$ were neutrophils. In addition, a $16.16 \mathrm{mg} / \mathrm{l}$ blood C-reactive protein was found to be above the normal range $(0-10 \mathrm{mg} / \mathrm{l})$. High erythrocyte sedimentation rate and D-dimer levels were also observed. The major pathogenesis of COVID-19 as a virus-directed respiratory infection was severe pneumonia.

\section{Normal - physiological values of NLR}

NLR values are calculated by dividing the absolute count of neutrophils by absolute count of lymphocytes. The normal values of NLR in adults are in the range bw. 1.0-2.3 (Yanti, 2016), median or mean values of NLR measured in healthy adult population is 1.85-2.2 (Azab et al, 2014, Forget et al, 2016, Holub et al, 2012). The grey zone of NLR are bw. 0.7-1.0 and 2.31-3.00. Pathological values of NLR are a) higher then 3.00 or b) lower then 0.7 NLR $<0.7$ (Tab.2).

\section{Use of neutrophil-to-lymphocyte ratio in viral infection}

Many research studies have tried to find biomarkers for the discrimination between bacterial and viral infection. Czech physicians assessed the potential use of the neutrophil to lymphocyte ratio (NLR) to discriminate between bacterial and viral infections. NLR was evaluated in 45 patients with bacterial infections: 24 patients with viral infections and 18 healthy adults. The medians of NLR were 11.73 in bacterial infections, 2.86 in viral infections and 1.86 in healthy controls. The NLCR cut-off value of $6.2 \mathrm{ex}-$ hibited a sensitivity value of 0.91 and a specificity value of 0.96 for bacterial infection. These results suggest a diagnostic potential for NLR to differentiate between viral (NLR $<6$ ), and bacterial infection - NLR higher then 6,2 (Holub et al, 2012). patients had clinically diagnosed infection, but not supported by microbiology and serology, and 29 patients had fever of non-infectious origin. They observed values NLR for bacterial infection mean 12.23, median 7.94 (4.5-15.0), and NLR for viral infection - mean 2.41, and median 0.63 (0.31-3.98), (Naess et al, 2017).

In uncomplicated viral infection the NLR may be in physiological range (1.0-2.4) or slightly elevated NLR bw. 2.4-4. Some viral infections (like monocytosis) when NLR is below 0.9 or even below 0.7 (lymphocytosis is higher than neutrophils count). Very low NLR $<0.7$ is common finding in viral infection, as well as in relative leukopenia with neutropenia, or in cancer patients after chemotherapy (Holub et al, 2012, Chalupa et al, 2011). We assume low NLR $<0.7$ as a pathological value. For bacterial infection are typical high NLR values higher than 7.0, or in bacteremia/septicemia higher then 11.0 or 17.0 (C. de Jager 2012, Loonen et al, 2014). We suggested a panel of four-parameters - biomarkers of inflammation to differentiate bacterial and viral infections: cytokine IL-6, C-reactive protein (CRP), procalcitonin (PCT), and neutrophil-to-lymphocyte ratio (Tab.3).

\section{Neutrophil-to-lymphocyte ratio for diagnosis ofCOVID-19}

The risk factors and clinical course of severe cases of COVID were analyzed on the cohort of 191 patients hospitalized in two hospitals in Wuhan (Fei Zhou et al,2020). The definitive diagnosis in each patient was put by confirmation of SARS-CoV-2 infection in respiratory specimens using sequencing or real-time RT-PCR methods. Routine blood examinations were complete blood count, coagulation profile, serum biochemical tests including lactate dehydrogenase, myocardial enzymes (hs-troponin), interleukin 6, serum ferritin, and procalcitonin. They have compared differences of laboratory markers between survivors (137 pts) and non-survivors (54 pts).In survivors were more frequent

Tab. 4. Valid clinical and laboratory markers for clinical stratification of COVID-19 infection ( Fei Zou et al, 2020).

\begin{tabular}{lccc}
\hline Parameter & $\begin{array}{c}\text { Survivors } \\
134 \mathrm{pts}\end{array}$ & $\begin{array}{c}\text { Non-survivors } \\
54 \mathrm{pts}\end{array}$ & Cut-off value \\
\hline Respiratory rate & $<24 \mathrm{br} / \mathrm{min}$ & $24 \mathrm{br} / \mathrm{min}$ & $24 \mathrm{br} / \mathrm{min}$ \\
\hline SOFA score & 1.0 & $4.5(4-6)$ & $3 \mathrm{points}$ \\
\hline Lymphocyte count $\mathrm{x} 10^{9}$ & $1.1 \times 10^{9} / \mu 1(1.0-1.5)$ & $0.610^{9} / \mu 1(0.5-0.8)$ & $0.8 \times 10^{9} / \mu 1$ \\
\hline LDH & $253 \mathrm{U} / \mathrm{L}$ & $512 \mathrm{U} / \mathrm{L}$ & $245 \mathrm{U} / \mathrm{L}$ \\
\hline hs-troponin I & $3.0(1.1-5.5)$ & $20.2(5.6-83)$ & $28 \mathrm{pg} / \mathrm{ml}$ \\
\hline D-dimer & $0.3-1.0 \mathrm{mg} / 1$ & $1.5-21 \mathrm{mg} / \mathrm{mg} / 1$ & $1.0 \mathrm{mg}$ \\
\hline Serum ferritin & $0.5 \mathrm{mg} / 1$ & $1.43 \mathrm{mg} / \mathrm{l}$ & $0.5 \mathrm{mg} / 1$ \\
\hline
\end{tabular}


Tab. 5. Cut-off values of predictive factors analysis of COVID-19 severity used in diagnostic tool Early warning score ( Song et al, 2020).

\begin{tabular}{lcc}
\hline Parameters/Cut-off & Cut-off value & AUROC \\
\hline T-score of pneumonia & 8.5 & 0.98 \\
\hline Age (years) & 44 & 0.74 \\
\hline NLR & 5.87 & 0.72 \\
\hline CRP mg/l & 14.22 & 0.73 \\
\hline ESR - sedimentation rate & 26.0 & 0.67 \\
\hline
\end{tabular}

leukopenia (20\% vs 9\%)and less leukocytosis higher then 10.0 $\mathrm{x} 10 \% / \mu \mathrm{l}(11 \%$ vs $46 \%)$.

In non-suvivors were typical significant lymphocytopenia median 0.6 thousand/ul $(0.5-0.8)$ when compared with less profound lymphopenia in survivors $1.1(0.8-1.5)$. Significant changes in hematological parameters in COVID-19 non-survivors are: anemia, lower platelets count, significant hypoalbuminemia $<29$ $\mathrm{g} / \mathrm{l}$, increased serum levels of lactate dehydrogenase (LDH), increase hs-troponin $\mathrm{I}$ - elevated $7 \mathrm{x}$ times more then in survivors. D-dimer levels were significantly higher in non-survivors mean $5.2(1.5-20 \mathrm{mg} / \mathrm{l})$ then in survivors mean value $0.6 \mathrm{mg} / 1(0.3-1.0)$. The optimal D-dimer cut-off value for stratification and prognosis is $1.0 \mathrm{mg} / \mathrm{L}$ (Tab. 4).

Very interesting laboratory findingin COVID-19 severe infection were the high concentrations of serum ferritin in nonsurvivors $1.43 \mathrm{mg} / \mathrm{L}$ versus $0.5 \mathrm{mg} / \mathrm{l}$ in survivors $(\mathrm{p}<0.001)$. Markers of bacterial infection PCT, CRP and cytokine IL- 6 were in normal range in COVID-19 survivors - observed low concentrations. Opposite to non-survivors all these markers were $\mathrm{mild} /$ moderate elevated, which may reflect bacterial infection and/or multiorgan dysfunction (Fei Zhouet al, 2020). The multiparametric approach for early diagnosis and laboratory monitoring of COVID-19 infection was suggested by Cong-Jing Song et al (2020). They constructed the diagnostic model using most valid parameters with significant impact on the clinical course, stratification according clinical severity and prognosis of severe COVID-19 infection.

After analysis of 1,311 patients with severe COVID infection, they found that signs of pneumonia on CT, fever, positive epidemiological anamnesis, values of neutrophil-to-lymphocyte ratio (NLR higher then 5.8), age (over 45 years old) and male sex were enrolled into COVID-19 early warning score (EWS), coined the term COVID-19 EWS (Tab. 5). The patients with COVID-19 infection had significant higher values of NLR 5.00 (IQR 2.3-13.9) than non-COVID patients NLR 2.7 (IQR 1.7-4.7, $\mathrm{p}<$ 0.001). Ill patients with COVID-19 infection had also profound lymphocytopenia, higher NLR ratio, lower platelet counts, higher erythrocyte sedimentation rate (ESR), mild elevated C-reactive protein a prokalcitonin, LDH, however cytokines like IL-2, IL4, IL-6, IL-10 and IFN-gamma were not elevated.

They established a novel and early-to-get early warnings core for COVID-19 screening: Probability COVID-19 $=2.79 \mathrm{x}$ fever $+4.58 \times$ history of close contact $+5.1 \times$ signs ofpneumonia on $\mathrm{CT}+0.97 \times \mathrm{NLR}+0.94 \mathrm{x} \operatorname{Tmax}+0.90 \mathrm{x}$ sex $+1.1 \mathrm{x}$ age. This scoring tool allows clinicians to more quickly and relatively ac- curetely detect COVID-19 patients, especially when the patient is asymptomatic and/or nucleic acid detection capacity of PCR is relatively lacking (Song et al, 2020). Liu et al (2020) followed the NLR values in adults with COVID-19 infection. They found out that age over 50 years and NLR values higher than 3.13 are associated with higher incidence of severe course of COVID-infection. In conclusion the NLR was the early identification of risk factors for COVID-19 severe illness. Patients with age over $50 \mathrm{y} / \mathrm{o}$ and NLR 3.13 facilitated severe illness, and should have rapidly access to the ICU if necessary (Liu et al, 2020). Recently published retrospective, multi-center, large sample study in the 43 settings from 10 provinces in China, in which 635 patients with COVID were enrolled (Ma et al, 2020). Of these, mild cases were 86 (14\%), ordinary cases $[486(76 \%)]$ and severe cases $[63(10 \%)]$, common symptoms at onset of disease were cough [356 (56\%)], fever and shortness of breath. An average of NLR of 635 patients was $4.04 \pm 4.68$, and elevated NLR were associated with the deterioration of clinical course [mild case (NLR $2.73 \pm 2.28$ ), ordinary/ moderate cases (NLR $3.58 \pm 3.07$ ), severe cases (NLR $9.38 \pm$ $10.52), \mathrm{p}<0.0001$. The area under the curve (AUC) of NLR was 0.727 and cut-off value was 4.06 , additionally, AUC of lymphocytes was 0.719 and cut-off value was 0.765 . NLR as inflammatory markers with rapid, convenient characteristics, NLR $\geq 2.22$ could be utilized as a predicting indicator for the early recognition COVID-19 and facilitate detection timely; meanwhile, NLR $\geq 4.06$ and lymphocytes count $\leq 0.765$ were as predicting indicator for severe COVID-19 (Ma et al, 2020).

\section{Conclusion}

Health and medical systems all over the world are in crude confrontation with COVID-19 Pandemia. Recently issued articles have analyzed demographic, clinical and laboratory parameters for early diagnosis and risk stratification (Zhou et al, 2020, Song et al, 2020). Complete blood count including white blood cell differential and calculated neutrophil-to-lymphocyte ratio seems to be helpful for screening and detection of novel corona virus infection.

A symptomatic and mild form of COVID-19 infecious disease are associated with normal leukocyte count and NLR, or even with leukopenia $<4.0 \times 10^{9} / \mu 1$ and lower NLR $<1.2$. Severe cases are coupled with leukocytosis and increased NLR values, higher than $\geq 3.13$ (Liu et al, 2020), or higher than $\geq 5.0$ (Song et al, 2020). Severe course of COVID-19 is associated with increased values of NLR and D-dimer during the first 14 days (Fu et al, 2020) and complicated with development of of severe acute respiratory syndrome (SARS). Typical clinical signs of SARS are dyspnoe, tachypnoe over 24 breath /min, severe hypoxemia and CT confirmed bilateral pneumonia (Zhou et al, 2020). Bad prognosis of COVID-19 is characterized by ongoing severe bilateral pneumonia, developing acute respiratory failure or ARDS with severe hypoxemia and very low oxygenation index $\mathrm{paO}_{2} / \mathrm{FiO}_{2}<150-100$ $\mathrm{mmHg}$, which should be treated by artificial mechanical ventilation. Other complications are bacterial super infection, circulatory shock, multiorgan dysfunction syndrome and even the death. 
466-470

Neutrophil - to - lymphocyte ratio is emerging stress and immune parameter which can be used alone or together with other biomarkers like D-dimer levels, serum ferritin, troponins and blood levels of CRP, PCT, IL-6, for screening, early diagnosis/detection and prognosis of COVID-19.

\section{References}

1. Azab B, Camacho-Rivera M, Taioli E. Average Values and Racial Differences of Neutrophil Lymphocyte Ratio among a Nationally Representative Sample of United States Subjects. PLoS One 2014; 9: e112361.

2. de Jager C, Wever PC, Gemen EPSA, Kuster R et al. ZThe neutrophil-lymphocyte count $\mathrm{z}$ ratio in patients with communitzy -acquired pneumonia. Plosd ONE 2012,7: e46561

3. Forget P, Khalifa C, Defour JP, Latinne D, Van Pel MC, De Kock M. What is the normal value of the neutrophil-to-lymphocyte ratio? BMC Research Notes 2017; 10:12.

4. Fu J, Kong J, Wang W, Wu M, Yao L et al. The Clinical Implication of dynamic Neutrophil to lymphocyte ratio and D-dimer in COVID-19: A retrospective study in Shuzhou China. Thrombosis Res 2020; 192: 3-8.

5. Holub M., Beran O, Kaspřiková L, Chalupa P: Neutrophil-to-lymphocyte count ratio as a biomarker of bacterial infection. Central EurJ Med 2012, 7: 258-261.

6. Chalupa P, Beran O, Herwald H, Kaspř́íková N, Holub M. Evaluation of potential biomarkers for the discrimination of bacterial and viral infections. Infection 2011; 39: 411-417.

7. Liu JY, Liu Y, Xiang P, Pu L, Xiong HF et al. Neutrophil-to-Lymphocyte ratio predicts severe illness patients with 2019 Novel Coronavirusin the Early stage. medRxiv 2020.

8. Kalelioglu T, Karamustafalioglu N. A new interpretation of neutrophil-lymphocyte ratio from psychiatric aspect. Bratisl Med J 2019; 120(8): 604-605.
9. Lee JS, Kim NY, Na SH, Youn YH, Shin CS. Reference values of neutrophil-lymphocyte ratio, lymphocyte-monocyte ratio, platelet-lymphocyte ratio, and mean platelet volume in healthy adults in South Korea. Medicine 2018; 97: e11138.

10. Loonen AJM, De Jager CPC, Tosserams J, Kostres R et al. Biomarkers and molecular analysis to improve blood stream infectzion diagnostics in an emergency care unit. PLoS One 2014, 9: e87315.

11. Ma Y, Shi N, Fan Y, Wang J et al. Predictive Value of Neutrophilto-lymphoyte ratio (NLR) for Diaghnosis and Worse Clinical Course of thr COVID-129: Findings from Ten Provinces of China. Lancet 2020,

12. Reiske L, Schmucker S, Pfaffinger B, Weler U, Steuber J, Stefanski V. Intravenous Infusion of Cortisol, Adrenaline, or Noradrenaline Alters Porcine Immune Cell Numbers and Promotes Innate over Adaptive Immune Functionality. J Immunol 2020.

13. Naess Are, Siri Daervold Nilssen, Reidun Mo, Gier EgilEide. Role of neutrophil to lymphocyte and monocyte to lymphocyte ratios in the diagnosis of bacterial infection in patients with fever. Infection 201745: 299-307.

14. Song CY, Xu J, He JQ, Qiang Lu YW. COVID-19 early warning core: a multiparameter screnogn tool to identify highly suspected patients. medRxiv 2020.

15. Yang W, Cao Q, Qin $\mathbf{L}$ et al. Clinical characteristics and imaging manifestations of the novel coronavirus disease (COVID-19): A multicenter study in Wenzhou city, Zhejiang, China.J Infect 2020 .

16. Zahorec R. Ratio of neutrophil to lymphocyte counts--rapid and simple parameter of systemic inflammation and stress in critically ill. Bratisl Lek Listy 2001; 102(1):5-14.

17. Zahorec R. Neutrophil-to-lymphocyte ratio. Sixteen-year long history since publication of our article in Bratislava Medical Journal. Bratisl Med J 2017;118 (6): 321-323.

18. Zhou F, Yu T, Du RH, Fan GH, Liu Y, LiuZB et al. Clinical course and risk factors for mortality of adult in patients with COVID-19 in Wuhan, Chine: a retrospective cohort study.Lancet March 92020.

Received March 20, 2020. Accepted April 4, 2020. 